https://helda.helsinki.fi

\title{
Quantum Theory and the Place of Mind in the Causal Order of Things
}

\section{Pylkkänen, Paavo}

Springer International Publishing AG

2019

Pylkkänen, P 2019 , Quantum Theory and the Place of Mind in the Causal Order of Things .

in Quanta and Mind : Essays on the Connection between Quantum Mechanics and the

Consciousness . Synthese Library (Studies in Epistemology, Logic, Methodology, and

Philosophy of Science) , vol. 414 , Springer International Publishing AG , pp. 163-171 . https://doi.org/10.1007/978-3

http://hdl.handle.net/10138/305675

https://doi.org/10.1007/978-3-030-21908-6_14

unspecified

acceptedVersion

Downloaded from Helda, University of Helsinki institutional repository.

This is an electronic reprint of the original article.

This reprint may differ from the original in pagination and typographic detail.

Please cite the original version. 


\title{
Quantum Theory and the Place of Mind in the Causal Order of Things
}

\author{
Paavo Pylkkänen \\ Department of Philosophy, History and Art Studies, University of Helsinki, Finland \\ and \\ Department of Cognitive Neuroscience and Philosophy, University of Skövde, Sweden \\ E-mail: paavo.pylkkanen@helsinki.fi \\ Web: https://philpapers.org/s/Paavo\%20Pylkkanen
}

\begin{abstract}
The received view in physicalist philosophy of mind assumes that causation can only take place at the physical domain and that the physical domain is causally closed. It is often thought that this leaves no room for mental states qua mental to have a causal influence upon the physical domain, leading to epiphenomenalism and the problem of mental causation. However, in recent philosophy of causation there has been growing interest in a line of thought that can be called causal anti-fundamentalism: causal notions cannot play a role in physics, because the fundamental laws of physics are radically different from causal laws. Causal anti-fundamentalism seems to challenge the received view in physicalist philosophy of mind and thus raises the possibility of there being genuine mental causation after all. This paper argues that while causal antifundamentalism provides a possible route to mental causation, we have reasons to think that it is incorrect. Does this mean that we have to accept the received view and give up the hope of genuine mental causation? I will suggest that the ontological interpretation of quantum theory provides us both with a view about the nature of causality in fundamental physics, as well as a view how genuine mental causation can be compatible with our fundamental (quantum) physical ontology.
\end{abstract}

\section{Introduction}

The received view in physicalist philosophy of mind states that causation can only take place at the physical level because everything that happens in the world is ultimately determined by the laws of physics and the physical domain is causally closed (cf. Sundström and Vassen 2017). This implies that non-physical entities cannot have any physical effects. If mind (whether conscious or unconscious) is taken to be non-physical (as is often done), then it cannot have any physical effects, and we end up with epiphenomenalism, the view that mental properties exist but have no causal influence upon the physical world. 
Epiphenomenalism is widely thought to be an unsatisfactory view and there have been many attempts to avoid it (see Robb and Heil 2018). In this paper I will discuss two ways to challenge the received view, and to save mental causation.

The first is provided by causal anti-fundamentalism, a view which states that causation is not part of the fundamental physical ontology of the world. If causal anti-fundamentalism is correct, then the received view is mistaken. Moreover, it has been suggested that causal anti-fundamentalism is compatible with the idea that fundamental physical facts ground higher-level causal facts, including those involving consciousness (Blanchard 2016: 256). Conscious experiences could then be seen as local events which determine events in their future.

A second way to challenge the received view is opened up by the ontological interpretation of quantum theory (Bohm and Hiley 1987, 1993). This interpretation suggests that the wave function in quantum theory describes a new type field which contains active information, which latter is a fundamental, causal factor organizing the motion of physical particles. Bohm (1990) proposed that by extending this quantum ontology in a natural way one can show how mental properties can influence matter. If Bohm's proposal is correct the received view is either mistaken, or else the concept "physical" in the received view needs to be extended to include active information and possibly mind/consciousness (which are traditionally often taken to be non-physical entities).

\section{Causal anti-fundamentalism}

Philosophers of mind and neuroscientists typically assume that we have a clear understanding of the nature of causality in the physical domain. But, in fact, there is a venerable tradition, dating back to Russell's (1913) causal antifundamentalism, arguing that causal notions can play no legitimate role in how physics represents the world (Frisch 2012; Price and Corry eds. 2007). Causal anti-fundamentalism has been succinctly summarized in a recent workshop description by Sundström and Vassen (2017): 
...causal notions cannot play a role in physics, because the fundamental laws of physics are radically different from causal laws. Causal laws typically describe how local events determine events in their future; for example, a causal law can connect smoking to later occurrences of cancer. By contrast, physical laws connect the entirety of physical reality in a time-symmetric manner: the entire state of the universe at a certain time equally determines the relative past and the future of the universe. It therefore appears reasonable to situate causation in the higher levels of science where local events are studied in a time-directed manner, such as biology and economy.

So, causal anti-fundamentalism is the thesis that causation is not part of the fundamental physical ontology of the world. If true, this entails that causation is not as central a feature of the world as ordinarily thought. But as was noted above, it has been suggested that causal anti-fundamentalism is compatible with the existence of causal facts as non-fundamental features of the world grounded in fundamental physical facts (Blanchard 2016: 256; cf. Cartwright 1979). I suggest that causal facts involving conscious experiences can also be seen as such features. This suggests a view in which conscious experiences can be seen as local events which determine events in their future (in a similar manner as with causal laws in special sciences such as biology and economy). Physics (and the causal closure of the physical domain) would no longer be an obstacle to mental causation. On the contrary: fundamental physical facts would now ground the causal facts involving conscious experiences! ${ }^{1}$

Let us summarize our discussion so far. According to the received physicalist view there is no room for mental causation, because all goings on in the world are ultimately determined by physics and physics itself is causally closed. In contrast, according to the view inspired by causal anti-fundamentalism the physical world is not governed by any fundamental relation of cause and effect.

\footnotetext{
1 Metaphysical grounding is, of course, a subtle topic in contemporary metaphysics, and we will not here enter into a discussion about what exactly it might mean when one says that fundamental physical facts ground causal facts. But see Bliss and Trogdon 2016. An additional challenge is to spell out how physical facts could ground non-physical, conscious facts (thanks to Tuomas Tahko for pointing out this challenge).
} 
But one can still hold that fundamental physical facts ground higher-level causal facts, including those involving consciousness. Conscious experiences can then be seen as local events which determine events in their future. Mental causation is possible!

Is this too good to be true? It is true that causal anti-fundamentalism, when suitably interpreted, gives us mental causation in some sense. But it also implies that causal facts (including those involving conscious experiences) are nonfundamental. One might worry whether this is too deflationist a view of (mental) causation. Also, causal anti-fundamentalism is admittedly weird, given the way we have traditionally been thinking about the laws of physics. Is it really the case that the physical world is not governed by any fundamental relation of cause and effect?

\section{Is causal anti-fundamentalism correct?}

Remember that causal anti-fundamentalism claims that the fundamental laws of physics are radically different from causal laws. Allegedly, one important difference is that while causal laws typically describe how local events determine events in their future, physical laws connect the entirety of physical reality. But do physical laws really connect the entirety of physical reality? In the spirit of scientific metaphysics, let us here consider how a physicist might answer the question. When discussing causality and chance in modern physics David Bohm proposed in 1957 that there is no known fundamental physical law that would be able to take into account the complete state of the world:

...every real causal relationship, which necessarily operates in a finite context, has been found to be subject to contingencies arising outside the context in question. (1957/1984: 3)

If Bohm is correct, perhaps the fundamental laws of physics are not radically different from causal laws, and there is causation in fundamental physics after all (for a brief discussion of Bohm's view, see Andersen et al. 2018). 
What about time-symmetry? Another reason why causal antifundamentalism claims that the fundamental laws of physics are radically different from causal laws has to do with time-symmetry. Causal laws typically describe how local events determine events in their future. But physical laws connect the entirety of physical reality in a time-symmetric manner. The idea is that the entire state of the universe at a certain time equally determines the relative past and the future of the universe.

Let as again consider the issue in the light of Bohm's thinking about physics. When Bohm in his 1951 text-book Quantum theory discusses time-symmetry he notes that classical theory is prescriptive and not causal. He points out, consistently with causal anti-fundamentalism, that in classical physics the idea of forces as causes of events became unnecessary and almost meaningless.

[this is so] because both the past and the future of the entire system are determined completely by the equations of motion of all the particles, coupled with their positions and velocities at any one instant of time. Thus, we can no more say that the future is caused by the past than we can say that the past is caused by the future. Instead, we say that the motion of all particles in space and time is prescribed by a set of rules, i.e. the differential equations of motion, which involve only these spacetime motions alone. (1951: 151)

But what about the quantum theory? Here Bohm makes a point which seems to challenge causal anti-fundamentalism in a profound way:

Whereas classical theory can be expressed in terms of a set of prescriptive rules relating space-time motions at different times, quantum theory cannot be so expressed. Energy and momentum (and therefore, the causal factors) cannot be eliminated in terms of velocities and positions of the component particles. The quantum theoretical concept of causality, therefore, differs from its classical counterpart. It must necessarily describe the relationships between space-time events as being "caused" by factors existing within matter (i.e., momenta). These are on the same fundamental and not further analyzable footing as that of space and time themselves. (1951:157) 
Bohm acknowledges that in quantum theory these causal factors control only a statistical trend in the course of space-time events. But he notes further that it is just this property of incomplete determinism that prevents the causal factors from becoming redundant. This, he says, gives a real content to the concept of causality in quantum theory.

So, if Bohm is correct, causal anti-fundamentalism is incorrect and there is causation in the physical world. This so, first of all, because each known fundamental physical law operates in a finite context, and secondly because in quantum theory, energy and momentum (and therefore, the causal factors) cannot be eliminated in terms of velocities and positions of the component particles (unlike in Newtonian physics).

Note, however, that what we have just reported above comes from Bohm's 1951 textbook Quantum theory where he was trying to explicate a version of the usual, "Copenhagen" interpretation (his explication is fairly similar to the approach of Wolfgang Pauli). But as is well known, Bohm himself published an alternative interpretation of quantum theory in 1952. This interpretation (or "the Bohm theory") assumed that an electron has simultaneously a well-defined position and velocity and is guided by a new type of field (mathematically described by the wave function $\Psi$ ). According to the usual interpretation of quantum theory, the wave function $\Psi$ does not describe an individual quantum system directly. Rather, $\Psi$ describes our knowledge of the quantum system to be observed (typically in terms of probabilities). In contrast, according to Bohm's 1952 theory, $\Psi$ describes an objectively real field, guiding a particle such as an electron.

What is the role of causality in the Bohm theory? A minimalist version of the theory (called "Bohmian mechanics") expresses quantum theory as a "firstorder" theory in terms of velocities (see Goldstein 2013). As far as I can judge, this is similar to classical physics in that causality (energy, momentum) can be eliminated if one wishes. In some versions of Bohmian mechanics the wave function is assumed to be law-like. So it seems that causality can be eliminated just as in classical physics!² However, Bohm and Hiley developed Bohm's 1952

\footnotetext{
${ }^{2}$ As I am not a physicist, I offer these proposals tentatively, to be discussed in more detail by those physicists specialized in Bohmian mechanics.
} 
theory into another direction ("the ontological interpretation"), where the role of the so called quantum potential energy is important. In this picture it may be possible to retain energy as irreducible and thus "genuine" causality in quantum mechanics. $^{3}$

The above suggests that there are certain ironies in the question quantum theory and causality. It seems that the usual interpretation of quantum theory implies that (statistical) causality is irreducible, ironically, because of the uncertainty principle. However, recent developments in the interpretation of quantum theory (e.g. Bohmian mechanics) may eliminate causality from fundamental physics (ironically, because of determinism, just as happened in Newtonian physics). But there is the possibility that in Bohm and Hiley's ontological interpretation, with its emphasis on quantum potential energy, we can retain genuine causality.

Let us now return to the issue of mental causation. We have noted that if Bohm and Hiley's interpretation is correct, and includes an irreducible form of quantum potential energy, then there is causation in the fundamental physical level and causal anti-fundamentalism is incorrect. But how can we then have mental causation? Doesn't the principle of the causal closure of the physical domain apply to the Bohm-Hiley scheme as well, thus leaving no room for mental properties qua mental to have an effect upon the physical?

Here I suggest that we consider Bohm and Hiley's (1993) suggestion that the ontological interpretation can be extended. So let us move on to examine the ontological interpretation and the way its extension might allow for mental causation.

\section{Extending the ontological interpretation of quantum theory}

According to the ontological interpretation of quantum theory quantum processes are guided by a field containing active information (described by the wave function $\Psi$, expressed in terms of the quantum potential). This involves a

\footnotetext{
${ }^{3}$ Again, I am offering this proposal tentatively as a philosopher, to be discussed in more detail by physicists.
} 
new type of causation which we may call informational causation. This information is radically holistic - it enables non-locality and irreducible objective wholeness of a many-body system.

It is important to realize that Bohmian active information is not Shannon information. The idea is that the form of the quantum field (described by the wave function) enfolds information about the environment (e.g. slits), and this information then literally IN-FORMS the movement of the particle through the quantum potential. The information is potentially active everywhere where the quantum potential non-zero, but actually active only where the particle is. Without such actual activity of "in-forming" the information would have no causal powers. So in this picture there needs to be actually active information for there to be any causally efficacious information at all.

Bohm himself (1990) proposed that active information can be seen as a "primitive mind-like quality" of elementary particles, suggesting a view that we may call Bohmian panprotopsychism (see Pylkkänen, forthcoming). The key principle here is that mental processes involve "activity of form" rather than "activity of substance". When you read the newspaper, you do not need to eat the paper, you abstract the form that is carried or enfolded in the movement of light waves. That form, when taken up by the nervous system and interpreted can give rise to a conscious experience of meaning of the information. Analogously, the electron is not pushed and pulled by the quantum wave. Rather, it is able to respond to the form of the quantum wave. It is "mind-like" in this sense. Note that Bohm thought it obvious that an electron is not (phenomenally) conscious (Bohm 1990). But we could ask whether the electron is in some sense "perceiving" (unconsciously) its environment via the quantum field. While many are still likely to see pan(proto)psychism as "a complete myth, a comforting piece of utter balderdash" (McGinn 2006: 93), it has become a subject of intense discussion in contemporary philosophy (see Strawson 2006; Goff et al. 2017; Pylkkänen, forthcoming).

How does mental causation work in Bohmian quantum ontology? Bohm suggested that it is natural to extend the quantum ontology. Just as there is a quantum field that informs the particle, there can be a super-quantum field that informs the first order quantum field, and so on (Bohm and Hiley 1993: 379- 
381). Let us further assume that the information contained in our mental states is a certain part of this hierarchy of fields. Through the hierarchy, mental states could then guide material processes, by reaching the quantum field of the particles and fields in the brain (Bohm 1990).

How exactly might such "quantum mental causation" work? There are currently a number of different proposals regarding how quantum effects might play a role in the neurophysiological processes underlying cognition and even consciousness (see Atmanspacher 2015; Pylkkänen 2018). From the perspective of the ontological interpretation the important question is whether there are some "quantum sites" in the brain where the quantum potential can have a nonnegligible effect. Hiley and Pylkkänen (2005) discuss one such possibility by applying the quantum potential approach to Beck and Eccles's (1992) ideas about the role of quantum mechanics in synaptic exocytosis. Beck and Eccles suggested that the appearance of low transition probabilities in synaptic exocytosis implies that there exists an activation barrier against the opening of an ion channel in the presynaptic vesicular grid. Hiley and Pylkkänen (2005: 212) suggested that it is the action of the quantum potential that effectively reduces the height of the potential barrier to increase the probability of exocytosis (this is an example of quantum tunneling). In the extended ontological interpretation the higher order "mental" fields can then in a natural way influence the quantum potential and in this way control synaptic exocytosis. Quantum tunneling in synaptic communication has recently been discussed in detail by Danko Georgiev (2018) and I suggest that a promising way to develop Bohmian quantum brain theory further is to examine Georgiev's proposals in the light of the richer picture of quantum processes that the ontological interpretation provides. Another possibility is to apply the Bohm scheme to recent proposals that quantum coherence is involved in ion channel conductivity and selectivity (Salari et al. 2017). By controlling the quantum potential in the ion channels, the higherorder mental fields might then be able to control the triggering of action potentials. Such speculations require a careful consideration of the problem of decoherence.

Alternatively, one might look in the light of the ontological interpretation at Penrose and Hameroff's proposal that consciousness depends on biologically 
"orchestrated" coherent quantum processes in collections of microtubules within brain neurons. Penrose and Hameroff (unlike Bohm and Hiley) give high importance to the role of the orchestrated objective reduction (collapse) of the quantum state in the regulation of axonal firings and the control of conscious behavior (see Hameroff and Penrose 2014). In the ontological interpretation such regulation would take place through the higher-order "mental" fields orchestrating the quantum potential (which need not involve collapse).

Let us summarize Bohmian mental causation. The Bohm-Hiley scheme gives a key causal role to information at the quantum level, by suggesting that information about the environment of the quantum particles is encoded in the wave function and guides the particles. It is reasonable to postulate a hierarchy of fields of information in complex systems such as brains. If free will and spontaneity is possible at the higher levels of information, then the hierarchy enables free will to guide physical action. The principle of the causal closure of the physical domain needs to be abandoned or else modified to include (traditionally non-physical) causal features such as information (for a more thorough discussion, see Pylkkänen 2007; 2017).

\section{Conclusion: two kinds of mental causation, contra the received view of physicalism}

In this paper we first considered how causal anti-fundamentalism suggests a possible route to mental causation. According to causal anti-fundamentalism the physical world is not governed by any fundamental relation of cause and effect. But it typically assumes that fundamental physical facts ground causal facts, including those involving consciousness. Conscious experiences can then be seen as local events which determine events in their future.

We also saw that there are reasons to think that causal anti-fundamentalism is incorrect. But we noted that there is another way to challenge the received view, namely by making use of the ontological interpretation of quantum theory. This interpretation suggests that information plays a fundamental causal role in the physical world. We argued that it is reasonable to assume that there exists a hierarchy of levels of information, with two-way causal influences between 
levels. Mental/conscious states are part of this hierarchy and can thus causally affect and be affected by physical processes at lower levels of the hierarchy.

In this short paper our discussion has been schematic, and many of the issues need to be explored more carefully in later research. For example, how to reconcile the suggestion that causality can be eliminated in classical physics, with the suggestion that it cannot be eliminated in (usual) quantum mechanics (and possibly in the Bohm-Hiley theory)? Further, the Bohm-Hiley theory is nonlocal, so what is the role of causality in those situations where a non-local quantum potential has a non-negligible effect (e.g. an EPR-type experiment; cf. Fenton-Glynn and Kroedel 2015; Walleczek 2016; Musser 2015)?

\section{Acknowledgements}

Earlier versions of this paper have been presented at the Annual Colloquium of the Philosophical Society of Finland in Helsinki, January 2018; at The Science of Consciousness conference in Tucson, USA, April 2018; at the Parmenides Foundation workshop "Rethinking Matter, Life and Mind", Tegernsee, Germany, September 2018; and at the "Scientific metaphysics" workshop at the University of Helsinki, January 2019. I thank the participants of these events for valuable comments and questions. The work for this paper was partially funded by the Fetzer Franklin Fund of the John E. Fetzer Memorial Trust.

\section{References}

Andersen, F., Anjum, R.L., and Mumford, S. (2018) Causation and Quantum Mechanics, in R.L. Anjum and S. Mumford What Tends to Be. The Philosophy of Dispositional Modality. London: Routledge.

Atmanspacher, H. (2015) Quantum Approaches to Consciousness, i (Summer 2015 Edition), E. N. Zalta (ed.), URL = http://plato.stanford.edu/archives/sum2015/entries/qt-consciousness/

Beck, F. and Eccles, J. (1992) Quantum aspects of brain activity and the role of consciousness. Proceedings of the National Academy of Sciences 89 (23): 11357-11361.

Blanchard, T. (2016) Physics and causation. Philosophy Compass 11, 256-266.

Bliss, R. and Trogdon, K. (2016) Metaphysical Grounding, The Stanford Encyclopedia of Philosophy (Winter 2016 Edition), E. N. Zalta (ed.), URL = https://plato.stanford.edu/archives/win2016/entries/grounding/ 
Bohm, D. (1951) Quantum theory. Englewood Cliffs NJ: Prentice-Hall. Dover edition 1989.

Bohm, D. (1957/1984) Causality and Chance in Modern Physics. London: Routledge.

Bohm, D. (1990) A new theory of the relationship of mind and matter. Philosophical Psychology 3, pp. 271-286.

Bohm, D. and Hiley, B. J. (1987) An Ontological Basis for Quantum Theory: I. Nonrelativistic Particle Systems, Physics Reports 144: 323-348.

Bohm. D. and Hiley, B.J. (1993) The Undivided Universe: An Ontological Interpretation of Quantum Theory. London: Routledge.

Cartwright, N. (1979) Causal laws and effective strategies. Nous 13, 419-37.

Fenton-Glynn, L. \& Kroedel, T. (2015). Relativity, quantum entanglement, counterfactuals and causation. British Journal for the Philosophy of Science 66 (1): 45-67.

Frisch, M. (2012) No place for causes? Causal skepticism in physics. Euro Jnl Phil Sci 2: 331-336.

Georgiev, D. (2018) Quantum Information and Consciousness: A Gentle Introduction. Boca Raton, FL: CRC Press.

Goff, P., Seager, W. and Allen-Hermanson, S. (2017) Panpsychism, The Stanford Encyclopedia of Philosophy (Winter 2017 Edition), E. N. Zalta (ed.), URL = https://plato.stanford.edu/archives/win2017/entries/panpsychism/

Goldstein, S. (2013) Bohmian Mechanics, The Stanford Encyclopedia of Philosophy, E. N. Zalta (ed.), URL = http://plato.stanford.edu/archives/spr2013/entries/qm-bohm/

Hameroff \& Penrose (2014) Consciousness in the universe: A review of the 'Orch Or' theory. Physics of Life Reviews 11 (2014), 39-78

Hiley, B.J. and Pylkkänen, P. (2005) Can mind affect matter via active information? Mind and Matter 3 (2): 7-26, URL = http://www.mindmatter.de/resources/pdf/hileywww.pdf

McGinn, C. (2006). Hard questions. Journal of Consciousness Studies 13 (10-11): 90-99.

Musser, G. (2015) Spooky Action at a Distance. New York: Farrar, Straus and Giroux.

Price, H. and Corry, R. (2007) eds. Causation, Physics, and the Constitution of Reality: Russell's Republic Revisited. Oxford: Clarendon Press.

Pylkkänen, P. (2007) Mind, Matter and the Implicate Order. Heidelberg and New York: Springer.

Pylkkänen, P. (2017) Is there room in quantum ontology for a genuine causal role of consciousness?, in A. Khrennikov \& Emmanuel Haven eds., The Palgrave Handbook of Quantum Models in Social Science. London: Palgrave Macmillan. 
Pylkkänen, P. (2018) Quantum Theories of Consciousness. In R. Gennaro (ed.) The Routledge Companion to Consciousness. London: Routledge.

Pylkkänen, P. (forthcoming) A quantum cure for panphobia. In W. Seager ed. Routledge Handbook of Panpsychism. London: Routledge.

Robb, D. and Heil, J. (2018) Mental Causation. The Stanford Encyclopedia of Philosophy, E. N. Zalta ed. (Winter 2018 Edition), E. N. Zalta (ed.), forthcoming URL = <https://plato.stanford.edu/archives/win2018/entries/mental-causation/>.

Russell, B. (1913) On the notion of cause. Proceedings of the Aristotelian Society 13, 1-26.

Salari, V., Naeij, H. \& Shafiee, A. (2017). Quantum interference and selectivity through biological ion channels. Scientific Reports 7: 41625.

Strawson, Galen (2006). 'Realistic monism - why physicalism entails panpsychism'. Journal of Consciousness Studies 13 (10-11): pp. 3-31.

Sundström, P. and Vassen B. (2017) Description of the "Where is there causation?" workshop, Umeå University, 27-29 October 2017, see https://philevents.org/event/show/34954

Walleczek, J. (2016) The Super-indeterminism in Orthodox Quantum Mechanics Does Not Implicate the Reality of Experimenter Free Will. J. Phys.: Conf. Ser. 701, 012005. 